\title{
Microscopic Findings Laterality
}

National Cancer Institute

\section{Source}

National Cancer Institute. Microscopic Findings Laterality. NCI Thesaurus. Code 6117594.

A qualifier for the side of the body the microscopic findings assessment is performed. 